\section{C.2 DIFFERENCES IN CARDIOVASCULAR RISK FACTOR PROFILES ACROSS OCCUPATIONAL GROUPS}

${ }^{1}$ Lucy A Barnes*, ${ }^{1}$ Marine Corbin, 'Hayley J Denison, ${ }^{1}$ Amanda Eng, ${ }^{1}$ Andrea 't Mannetje,

2,3Stephen Haslett, 'David McLean, 'Lis Ellison-Loschmann, ${ }^{4}$ Rod Jackson, 'Jeroen Douwes.

${ }^{1}$ Centre for Public Health Research, Massey University, Wellington, New Zealand; ${ }^{2}$ Institute of Fundamental Sciences - Statistics, College of Sciences, Massey University, Palmerston North, New Zealand; ${ }^{3}$ Statistical Consulting Unit, The Australian National University, Acton, Australia; ${ }^{4}$ Section of Epidemiology and Biostatistics, School of Population Health, Faculty of Medical and Health Sciences, The University of Auckland, Auckland, New Zealand

\subsection{6/OEM-2019-EPI.150}

Background Differences in cardiovascular disease (CVD) risk between occupations have been observed, but what specific risk factors underlie these patterns remains unclear. This study assessed how established CVD risk factors differ across occupational groups in New Zealand, and whether patterns differ by gender and ethnicity.

Methods Two probability-based sample surveys of the general New Zealand adult population $(2004-2006$; $n=3003)$ and of the indigenous peoples of New Zealand (Māori; 2009-2010; $\mathrm{n}=2107$ ), for which occupational histories and lifestyle factors were collected, were linked with routinely collected health data. For males and females in each cohort, smoking, body mass index, diabetes, hypertension and high cholesterol were dichotomised and compared between occupational groups using age-adjusted logistic regression. In addition, two way tables and chi-squared tests were used to compare the distribution of risk factors between white and blue-collar workers.

Results The prevalence of ever smoking was greater in bluecollar compared to white-collar workers of both cohorts, i.e. general population male $(56 \% ; 47 \%)$, general population female $(62 \% ; 47 \%)$, Māori male $(68 \% ; 55 \%)$ and Māori female $(84 \% ; 64 \%)$. For diabetes and high cholesterol, differences were statistically significant only for general population females (11\%; 6\% and 26\%; 16\% respectively). Hypertension prevalence was higher in blue versus white-collar workers for general population females (36\%; 29\%), Māori males (43\%; $36 \%$ ) and Māori females (44\%; 37\%), but not for general population males $(35 \% ; 34 \%)$. Results by occupational group revealed the same patterns with some noteworthy exceptions. In both cohorts, clerks had an increased risk of hypertension, particularly among males. Male agricultural workers in the general population cohort had a reduced risk of hypertension, diabetes and high cholesterol, but this pattern was not observed for females or among Māori.

Conclusion This study showed differences in profiles of known CVD risk factors across occupations, with some notable ethnic and gender differences.

\section{C.3 THE ASSOCIATION BETWEEN LONG-TERM EXPOSURE FROM HEAVY PHYSICAL WORK LOAD AND CORONARY HEART DISEASE IN MIDDLE AGED MEN}

\footnotetext{
1,2Tomas Hemmingsson*, ${ }^{2}$ Jenny Selander, ${ }^{2}$ Katarina Kjellberg. ${ }^{1}$ Stockholm University, Stockholm, Sweden; ${ }^{2}$ Karolinska Institutet, Stockholm, Sweden
}

\subsection{6/OEM-2019-EPI.151}

Objectives Previous studies have shown conflicting results concerning the association between heavy physical work load and risk of coronary heart disease (CHD). The aim of this study was to investigate the association between long-term exposure to heavy physical work load in middle age and risk of CHD among Swedish men during almost 20 years of follow-up.

Methods The study is based on a cohort of around 40000 Swedish males, born 1949-51, with census information on occupation in 1985 and 1990 (at ages 34-41). Aspects of physical workload was estimated from job exposure matrices based on questions concerning heavy lifting, strenuous work postures, and physically strenuous work from the Swedish Work Environment Surveys 1989-97. Mean values for each physical exposure variable were assigned to over 300 occupational titles from the censuses conducted in 1985 and 1990. Only those 29530 subjects who were in the same quintile of exposure both years were included in the analyses. Information on smoking behavior and body mass index (BMI) was collected at compulsory conscription for military training in $1969 / 70$ (at 18-20 years of age), and follow up data on CHD (1457 cases) between the years 1991-2009.

Results An increased relative risk of CHD were found among workers in the quintiles with medium low $(\mathrm{HR}=1.36$ $\mathrm{CI} 95 \%=1.14-1.62)$, medium $(\mathrm{HR}=1.46 \mathrm{CI} 95 \%=1.23-1.74)$, medium-high $\quad(\mathrm{HR}=1.62 \mathrm{CI} 95 \%=1.36-1.91)$, and high $(\mathrm{HR}=1.45 \mathrm{CI} 95 \%=1.22-1.71)$ exposure to heavy lifting at work compared with those in the quintile with low exposure to heavy lifting. After adjustment for smoking and BMI the risk estimates were somewhat lower but still significantly increased. Risk estimates of the same magnitude were seen for a number of other aspects of the physical work environment.

Conclusion There was an association between long-term exposure from heavy physical work load and CHD even after adjustement for smoking and BMI.

\section{C.4 THE RELATIONSHIP BETWEEN THE DEMAND-CONTROL MODEL AND INCIDENT CARDIOVASCULAR DISEASE IN ONTARIO CANADA. A LINKED ANALYSIS OF 12,358 WORKERS}

Peter Smith*. Institute for Work and Health, Toronto, Canada

\subsection{6/OEM-2019-EPI.152}

Dimensions of the demand-control psychosocial work environment model have previously been linked increased incidence of both diabetes and hypertension in Canada. However, few investigations in Canada have examined the relationship between the demand-control model and cardiovascular disease.

We linked Ontario respondents to the 2000, 2002 and 2003 cycles of the Canadian Community Health Survey, to administrative health care and hospitalisation databases to identify incident cases of cardiovascular disease over a 15 years follow-up period. Our sample included employed labour market respondents, aged 35 and older, working 15 or more hours per week, with information on the psychosocial work environment, and who were free of heart disease at baseline $(n=12,358)$. The demand-control model was defined based on median splits (active, passive, low strain and high strain), although models using the mid-population approach and the ratio of control to demands were also examined.

Over the study period 507 incident cardiovascular events occurred. The incidence was higher among men (5.5\%) compared to women $(2.6 \%)$. No statistically significant differences 
were observed across the four different demand-control categories in the full sample. In stratified analysis, passive work environments (low control and low demands) and low-strain environments (high control and low demands) were associated with increased risk of heart disease $(\mathrm{HR}=1.94$, 95\% CI 0.99 3.81; $\mathrm{HR}=2.06$, 95\% CI 1.01-4.17), compared to active work (high demands and high control) among women. No similar relationship was observed among men.

Among a representative sample of Ontario workers, high strain work environments were not associated with increased risk of heart disease. Passive and low strain environments (both characterised by low psychological demands) were associated with increased risk of heart disease among women, but not men. Implications of these findings for assessment of the psychosocial work environment in Canada will be discussed.

\section{Biomarkers of Exposure}

\section{D.1 INFLAMMATORY MARKERS IN THE PLASMA OF FIREFIGHTERS HEAVILY EXPOSED TO PARTICULATES}

Jean-Michel Galarneau*, Nicola Cherry. University of Alberta, Edmonton, Canada

\subsection{6/OEM-2019-EPI.153}

Introduction In 2016 firefighters from Alberta, Canada deployed to a catastrophic fire in Fort McMurray. In the first few days, firefighters experienced heavy smoke exposures during greatly extended work shifts. Blood samples were collected to determine whether inflammatory markers might constitute a useful biomarker of exposure. In first responders exposed during the Word Trade Center disaster, inflammatory markers in serum samples collected within 6 months post-event were associated with poor recovery from exposure-related lung disorders.

Methods Blood samples were collected at two fire services. At Service A, first samples were drawn two weeks from the start of the fire and second samples after 3-4 months. At Service B samples were collected over 4 weeks, starting 4 months from the first exposure. Samples were immediately centrifuged and the plasma stored at $-80 \mathrm{C}$ before being evaluated for 42 cytokines or chemokines using a multiplex assay. A principal component analysis was carried out to reduce the number of correlated outcomes. Exposure to particulates was estimated for each firefighter using environmental PM2.5, total hours exposed, tasks carried out and the use of respiratory protection. Respiratory symptoms immediately before the fire, immediately post fire and at 4 months were collected using visual analogue (VA) scales.

Results Inflammatory markers were assayed for 242 plasma samples from 175 firefighters. Six components were extracted of which only one, labelled the inflammatory marker component $(\mathrm{IMC})$ was related to estimated exposure $(\mathrm{p}<0.001)$ : values decreased with time since last exposure $(p<0.001)$. All respiratory symptoms post-fire were greater in those with higher estimated PM2.5. IMC scores were independently related to cough and wheeze at 4 months, but the biomarker did not contribute to models for these endpoints that also included PM2.5.

Conclusions Inflammatory markers were related to exposure but did not improve prediction of symptoms in the first months post fire.

\section{D.2 EVIDENCE OF DNA METHYLATION CHANGES BY CARBON NANOTUBES IN A TRANSLATIONAL STUDY DESIGN}

${ }^{1}$ Manosij Ghosh*, 'Deniz Öner, ${ }^{1,2}$ Lode Godderis, 'Peter Hoet. ${ }^{1} \mathrm{KU}$ Leuven, Department of Public Health and Primary Care, Centre Environment and Health, Leuven, Belgium; ${ }^{2}$ dewe, External Service for Prevention and Protection at Work, B-3001, Heverlee, Belgium

\subsection{6/OEM-2019-EPI.154}

Introduction While studies have addressed genotoxic effects of CNT, only limited information are available on epigenetic effects. We designed a study to investigate DNA methylation alterations in vitro, in vivo and in occupationally exposed workers.

Material and methods In vitro studies were performed in 16$\mathrm{HBE}$ and THP-1 cells. For the in vivo study, BALB/c mice were administered intratracheally with single-wall CNT (SWCNTs) and multi-wall (MWCNTs) at high $(2.5 \mathrm{mg} / \mathrm{kg})$ and low $(0.25 \mathrm{mg} / \mathrm{kg})$ doses. For the cross sectional study, 24 workers exposed to aggregates of MWCNT of $500 \mathrm{~nm}-$ $100 \mu \mathrm{m}$ with concentrations of $4.6-42.6 \mu \mathrm{g} / \mathrm{m} 3$ and 43 unexposed referents were recruited. Global DNA methylation and demethylation patterns were analysed by LC-MS/MS. Methylation of specific genes was measured by Pyromark $24^{\circledR}$ (Qiagen). Genome-wide assessment of DNA methylation was performed with Infinium HumanMethylation450 BeadChip Array.

Results In general, we did not find global DNA methylation alteration for both CNTs. In 16-HBE cells, differentially methylated and expressed genes (MWCNTs $>$ SWCNTs) from p53 signalling, DNA damage repair and cell cycle pathways were observed. In THP-1 cells, CNTs induced promoter-specific methylation of genes involved in several signaling cascade, vascular endothelial growth factor and platelet activation pathways. In lungs of BALB/c mice CNTs affected methylation of ATM gene. Finally, analysis of gene-specific DNA methylation in exposed workers revealed significant changes for DNMT1, ATM, SKI, and HDAC4 promoter CpGs.

Conclusions Epigenetic changes seem to occur at sub cyto-genotoxic concentrations in vitro. Alteration in DNA methylation pattern could be a natural reaction of cells but could also silence critical genes and reprogram cellular functions.

\section{O6D.3 EVALUATION OF POLYCYCLIC AROMATIC HYDROCARBONS EXPOSURE ACROSS OCCUPATIONS IN KOREA USING URINARY METABOLITE 1- HYDROXYPYRENE}

${ }^{1}$ Dong-Hee Koh*, ${ }^{2}$ Ju-Hyun Park, ${ }^{3}$ Sang-Gil Lee, ${ }^{4}$ Hwan-Cheol Kim, ${ }^{5}$ Sang-Jun Choi, ${ }^{1}$ Hyejung Jung, ${ }^{6}$ Dong-Uk Park. 'Department of Occupational and Environmental Medicine. Int St. Mary Hospital, Incheon, South Korea; ${ }^{2}$ Department of Statistics, Dongguk University, Seoul, South Korea; ${ }^{3}$ Occupational Safety and Health Research Institute, Korea Occupational Safety and Health Agency, UIsan, South Korea; ${ }^{4}$ Department of Occupational and Environmental Medicine, Inha University, Incheon, South Korea; ${ }^{5}$ Department of Occupational Health, Daegu Catholic University, Daegu, South Korea; ${ }^{6}$ Department of Environmental Health, Korea National Open University, Seoul, South Korea

\subsection{6/OEM-2019-EPI.155}

Objectives Polycyclic aromatic hydrocarbons are a wellknown carcinogen causing lung and skin cancers in exposed workers. Several occupations such as coke production have been reported to be associated with high PAHs exposure. However, previous reports have been 